\title{
Dynamic Analysis of the STARC-ABL Propulsion System
}

\author{
Jonathan L. Kratz ${ }^{1}$, and George L. Thomas ${ }^{2}$ \\ NASA Glenn Research Center, Cleveland, Ohio, 44135
}

\begin{abstract}
In the pursuit of Electrified Aircraft Propulsion (EAP), much of the attention is on the development of hybrid electric concept vehicles and their propulsion systems from a steady-state performance perspective. While it is steady-state performance that largely determines the efficiency of civil air transports, engine operability and transient performance define constraints for the steady-state design that impact efficiency and system viability. Neglecting dynamics and control technologies can result in an over-designed, sub-optimal propulsion system or a concept that is not feasible. Thus, dynamic system studies were conducted on the propulsion system of the conceptual aircraft design known as the Singleaisle Turboelectric AiRCraft with Aft Boundary Layer propulsor (STARC-ABL). This paper describes the development of a controller to verify the baseline concept's feasibility from an operability perspective. Further, studies were conducted to identify excessive stability margin in the baseline design that could be traded for potential benefits in efficiency through an engine re-design. This study revealed the potential to reduce the high pressure compressor (HPC) stall margin by $3 \%$. Finally, a study was conducted to investigate the potential benefit of adding energy storage to the STARC-ABL concept that further improves operability and enables more gains in engine efficiency and performance. The energy storage provided an additional $0.5 \%$ stall margin can be removed from the HPC.
\end{abstract}

\section{Nomenclature}

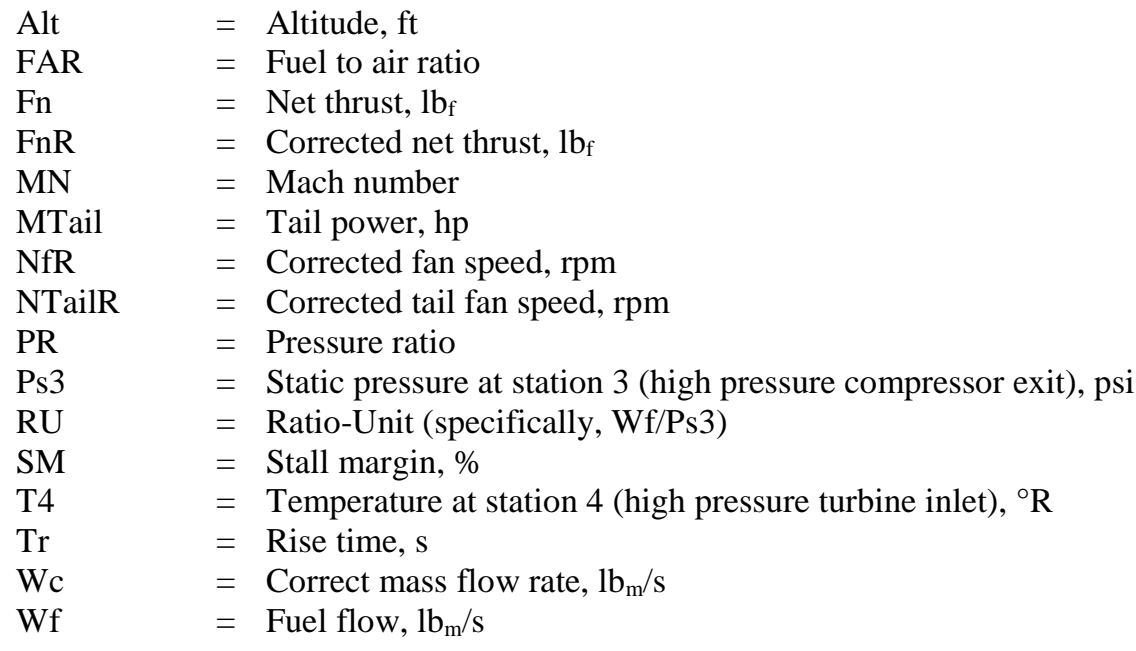

\section{Introduction}

THE electrification of aircraft brings a number of new potential benefits that include improved system efficiency, reduced noise and emissions, and the enabling of new capabilities [1,2]. However, these benefits are only

\footnotetext{
${ }^{1}$ Research Engineer, 21000 Brookpark Rd., Cleveland OH, MS-77-1, AIAA Member. jonathan.kratz@nasa.gov

${ }^{2}$ Research Engineer, 21000 Brookpark Rd., Cleveland OH, MS-301-5, AIAA Member. george.l.thomas@ nasa.gov
} 
realizable if the concepts are feasible from an operability perspective. Furthermore, the capability of controls should be considered in the system design to avoid over designing the engine at the expense of efficiency and performance. This is particularly true as the electrification of the aircraft propulsion system introduces new actuators and degrees of freedom that could be used by the control system to produce benefits for the turbomachinery and aircraft [3]. To this end, the National Aeronautics and Space Administration (NASA) turboelectric conceptual vehicle and propulsion system known as the Single-aisle Turboelectric AiRCraft with Aft Boundary Layer propulsor (STARC-ABL) [4,5] was evaluated from a dynamic operability perspective.

This study built on previous work $[6,7,8]$ that evaluated the feasibility of advanced engine concepts from a dynamic operability perspective and identified excess operability margin within those designs. The excess margin expands the engine design space to enable more efficient designs. Ref. [6] evaluated an advanced geared turbofan that features a compact gas turbine and variable area fan nozzle. Ref. [7] evaluated a prospective propulsion system for the Subsonic Ultra Green Aircraft Research (SUGAR) Volt parallel hybrid electric aircraft concept known as the hFan. Dynamic system analysis techniques developed in Ref. [6] and [7] were leveraged in the evaluation of the STARC-ABL concept. The feasibility of the STARC-ABL propulsion system concept was evaluated on the basis of dynamic operability under nominal operating conditions. Off-nominal operating modes that may arise from faults were outside of the scope of this study. The propulsion system was verified to operate within its defined operating limits and maintain sufficient stall margin in its compression elements throughout the entire flight envelope while undergoing transient maneuvers. In addition to the feasibility assessment, excessive operability margins were identified in an effort to expand the engine design space. Finally, a potential use of energy storage to promote operability of the STARC-ABL propulsion system was investigated to further expand the engine design space.

The rest of the paper is organized as follows. Section II provides a description of the STARC-ABL system and its propulsion system model. Section III discusses the development of the controller design and evaluates the feasibility of the concept from an operability perspective. Section IV applies dynamic system analysis (DSA) tools to evaluate the performance vs. operability trade in an effort to identify excessive operability margin that enables a more efficient engine design. This also includes a short consideration of the impact energy storage could have on the operability of the system. Finally, Section V provides some concluding remarks.

\section{NASA STARC-ABL Engine Model Definition}

STARC-ABL is a single-aisle tube and wing commercial transport concept similar in appearance to a modern Boeing 737. The main difference is that it has a ducted, electrically driven, boundary layer ingesting tail-cone thruster. The idea is to energize the low momentum airflow at the rear of the fuselage to produce thrust and reduce drag. The STARC-ABL concept is projected to reduce fuel consumption in reference to a traditional single-aisle tube and wing design with underwing engines that possess the same level of technology [4]. Since STARC-ABL is a turboelectric concept, there is no energy storage present to absorb or supply power and thus all of the power needed by the tail-cone fan must be immediately supplied by the underwing engines. The underwing engines are geared turbofans with $\mathrm{N}+3$ technologies, implying their availability in the 2030-2035 time-frame. The entire propulsion system is capable of producing a little over $40,000 \mathrm{lb}_{\mathrm{f}}$ of thrust at the sea level static (SLS) condition. The propulsion system has two underwing two spool engines that each have a geared fan, compact gas turbine, and variable area fan nozzle (VAFN). Each engine has a $2000 \mathrm{hp}$ generator coupled to the low pressure spool that is used to extract power for the tail-cone thruster driven by a $3500 \mathrm{hp}$ motor. The electrical power is transmitted via a $1000 \mathrm{~V}$ direct current bus. Figure 1 and 2 give a high-level representation of the STARC-ABL concept and propulsion system. Figure 3 and 4 are more detailed representations of the underwing engines and tail-cone thruster respectively. They define the geometry of the turbomachinery and indicate the presence of the electrical machines. These images were created by the Numerical Propulsion System Simulation (NPSS) [10] Weight Analysis of Turbine Engines (WATE++) [11] code.

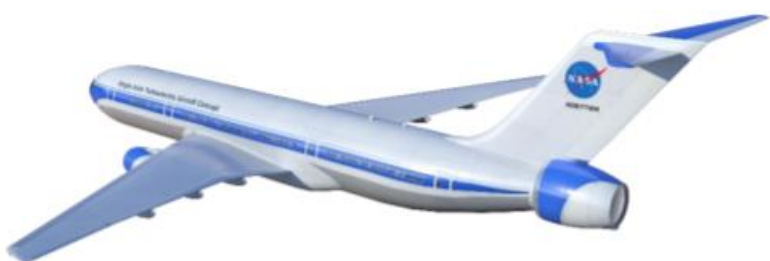

Fig. 1 Artistic rendition of STARC-ABL [9]

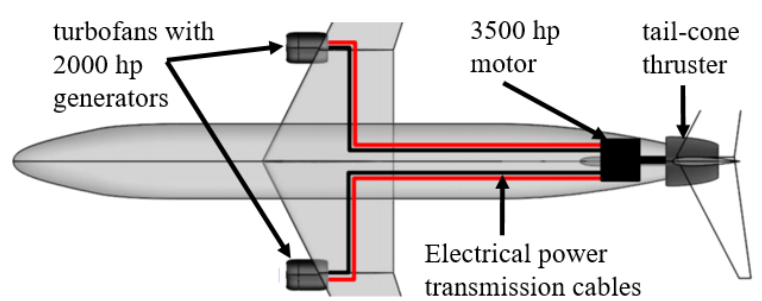

Fig. 2 High-level representation of STARC-ABL

[9] 
The conceptual STARC-ABL propulsion system was modeled using the NPSS code [12]. The turbomachinery was modeled with performance maps that define the relationships between rotational speed, pressure ratio, mass flow rate, and efficiency. The model was originally developed to run at steady-state operating points. The model was then modified to enable dynamic operation of the engine and was migrated to the MATLAB/Simulink ${ }^{\circ}$ environment via the S-function [13] interface to facilitate dynamic analysis and control design. The S-Function interface allowed the

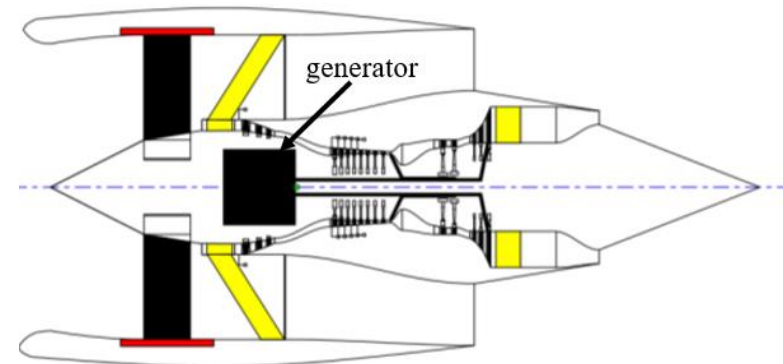

Fig. 3 Schematic of the underwing engines produced by NPSS WATE++ code [9]

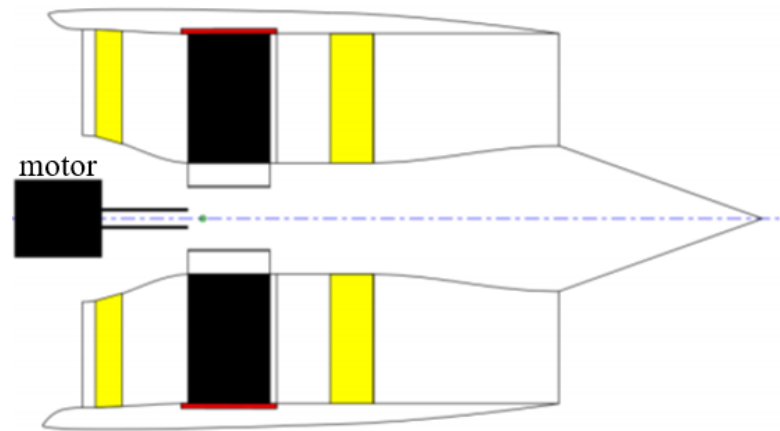

Fig. 4 Schematic of the tail-cone propulsor produced by NPSS WATE++ code [9] NPSS engine model to run within Simulink, with various model inputs being controlled by Simulink. The S-Function returns outputs back to Simulink. A closed-loop controller was constructed in the Simulink environment, providing inputs to and receiving feedback from the NPSS S-function.

The underwing engines have four main control actuators: a fuel metering valve (FMV) that controls the fuel flow, Wf, supplied to the combustor to achieve the desired thrust or power; a VAFN that modifies the area of the fan nozzle to control pressure rise and maintain fan operability; a variable bleed valve (VBV) that can increase the flow through the low pressure compressor (LPC) in order to maintain LPC operability; and the Variable Stator Vanes (VSV) that modify flow incidence angle within the first several stages of the high pressure compressor (HPC) to ensure HPC operability over its range. Of these control inputs, the fuel flow was the only one that was actively controlled. The other inputs were scheduled based on the given flight condition and power condition. Off-nominal operation of the VAFN and VBV were modeled, while the nominal effects of the VSV were incorporated in the HPC performance map. The FMV, VAFN, and VBV were given first order dynamics similar to the modeling approach implemented in Ref. [14]. The fuel controllers developed in this effort used net thrust, Fn, as the control variable implying direct control of thrust. The commanded thrust was provided by a normalized thrust command or corrected thrust, FnR. While direct thrust control is not feasible in an actual application, it was appropriate for this effort that seeks to broadly assess potential capabilities rather than detail a realistic controller implementation.

STARC-ABL is a turboelectric propulsion concept, which implies that energy that is extracted from the underwing engines is immediately transferred to and applied by the tail-cone motor. In the model, the engines were assumed to operate at the same power level and each generator supplied half of the requested power to the motor. The power was scheduled based on the current flight condition, which was described by the Mach number (MN), altitude (Alt), and corrected thrust. The electrical system was assumed to consist of the electric machines with $96 \%$ efficiency, inverters with $98 \%$ efficiency, and cables with $99.6 \%$ efficiency. The components were modeled simplistically by applying some efficiency losses resulting in an overall power transmission efficiency of $\sim 90 \%$. First order dynamics were applied to the tail fan motor to model delays in the power transmission and the electric machines. The tail fan has a VAFN similar to the underwing engines that serves the purpose of promoting operability of the tail fan.

The VBV, turbofan VAFN, tail VAFN, and motor power schedules were derived from numerous steady-state operating points and are correlated to the flight condition described by the MN, Alt, and corrected fan speed. The turbofan actuators used the corrected fan speed of the turbofan, NfR. The tail VAFN used the corrected fan speed of the tail fan, NTailR.

The propulsion system model was modified to include component health parameters that were prescribed according to the deterioration level of the propulsion system, ranging from new to end-of-life (EOL). Note that all controllers were designed for a mid-life propulsion system. The health parameters included flow, efficiency, and pressure ratio modifiers for each of the turbomachinery components as applicable. The deterioration model used to determine the range and rate of deterioration was adapted from the model applied in the Commercial Modular AeroPropulsion System Simulation 40,000lb $\mathrm{f}_{\mathrm{f}}(\mathrm{C}-\mathrm{MAPSS} 40 \mathrm{k})$ engine model [14]. 


\section{Controller Development \& Feasibility Assessment}

This section details the development and analysis of a controller for the STARC-ABL propulsion system. This effort involved developing a full flight envelope controller as well as making some modifications to the VAFN schedules.

\section{A. VAFN Area Constraints}

In the original STARC-ABL model, the VAFN area was not constrained for the turbofans or tail fan. The area of the turbofan and tail fan nozzles could range from $1250-5560 \mathrm{in}^{2}$ and $1800-6080 \mathrm{in}^{2}$ respectively, or $78 \%$ and $70 \%$ of their maximum nozzle areas. Thus, an effort was made to limit the range of area variation to improve VAFN responsiveness and reduce the likelihood of fan stall. The approach taken was to run the engine model at various steady-state points throughout the flight envelope and to reduce the VAFN where applicable while still respecting operability limits. This required maintaining fan, and tail fan stall margins (SMs) above 10\%. The updated turbofan and tail fan VAFN schedules limited the areas between $1750-3150$ in $^{2}$ and $2425-4400$ in $^{2}$ respectively, or about $45 \%$ of the maximum nozzle area in both cases. While this change in operation of the tail fan VAFN may affect the boundary layer ingestion model embedded in the NPSS model, this detail was ignored in the analysis. The tail fan VAFN schedule is only effected at a subset of flight conditions, particularly at low power and at low altitudes and Mach numbers. At low Mach numbers, the boundary layer is relatively small and the effects of boundary layer ingestion are much less prominent. Thus, the impact on the boundary layer ingestion model at these conditions is expected to be small. The tail fan VAFN schedule is not effected for much of the propulsions systems operating range, including at cruise conditions. Therefore, the effect on overall performance is expected to be insignificant. There may be some flight conditions, particularly during descent, in which the restricted VAFN area could impact performance. The reduction in tail fan VAFN area at these flight conditions would be expected to reduce the amount of boundary layer ingested by the fan, thus reducing the thrust produced by the tail fan and increasing the pressure drag on the aft portion of the fuselage. Without updating the boundary layer ingestion model, these small changes in performance will not be captured correctly. Ideally, the boundary layer ingestion model would be updated to reflect the impacts of placing minimum and maximum limits on the tail fan VAFN area. However, given the small impact on overall performance and the complexities associated with coupling these models, it was outside of the scope of this study. With these caveats, the substantial reduction in maximum nozzle area and variation in nozzle area demonstrated here, promotes the feasibility of the concept.

\section{B. Controller Design}

Similar to previous work [6,7,8], the open-source Tool for Turbine Engine Closed-loop Transient Analysis (TTECTrA) controller architecture and design tools were used to design a controller for the STARC-ABL propulsion system. The TTECTrA tool was used to create a full flight envelope, single-input single-output, gain-scheduled proportional integral (PI) controller with integral wind-up protection (IWP). The controller input is the fuel flow rate while the control variable is the net thrust. The set-point thrust is determined by the corrected thrust command. The corrected thrust is the thrust normalized on a scale between 0 and 1 where 0 corresponds to the minimum thrust the engine will produce at the current flight condition (Mach number and altitude), and 1 corresponds to the maximum thrust the engine will produce at that same point in the flight envelope. Thus, the set-point thrust was determined by first interpolating the maximum and minimum thrust at the current MN and Alt using data tables and then using the commanded FnR to compute the dimensional thrust value. The thrust returned by the engine model is compared with the command to compute the error that enters the controller. Note that thrust control is normally accomplished indirectly by controlling a variable that has a strong correlation with the engine's thrust, such as the corrected fan speed or engine pressure ratio. While direct thrust control is not used on engines today, it may become common practice in the future using onboard nonlinear engine models and model-based engine control [15]. Regardless, direct control of thrust simplifies the simulation study and still accomplishes the end goal. Figure 5 is a schematic of the overall structure of the thrust/power control logic for the STARC-ABL propulsion system. Note that because Fig. 5 only addresses thrust/power management, it excludes the scheduled control logic for the VBV, and VAFNs.

Power management of the engine was dealt with by the fuel controller. A major piece of that was the gain-scheduled PI controller mentioned prior. However, this was not sufficient. Limit logic must be present to prevent the engine from encountering potentially hazardous conditions that unnecessarily jeopardize the safety of the aircraft or the health of the engine. This includes preventing the engine from stalling, combustor blow-out, over-speed, over-temperature, etc. The fuel flow controller used a modified TTECTrA control architecture that included several limit controllers and a max-min logic to determine which controller was active. Limit controllers included a maximum ratio-unit (RU) limiter, a maximum high pressure turbine inlet temperature (T4) limiter, a minimum RU limiter, and 


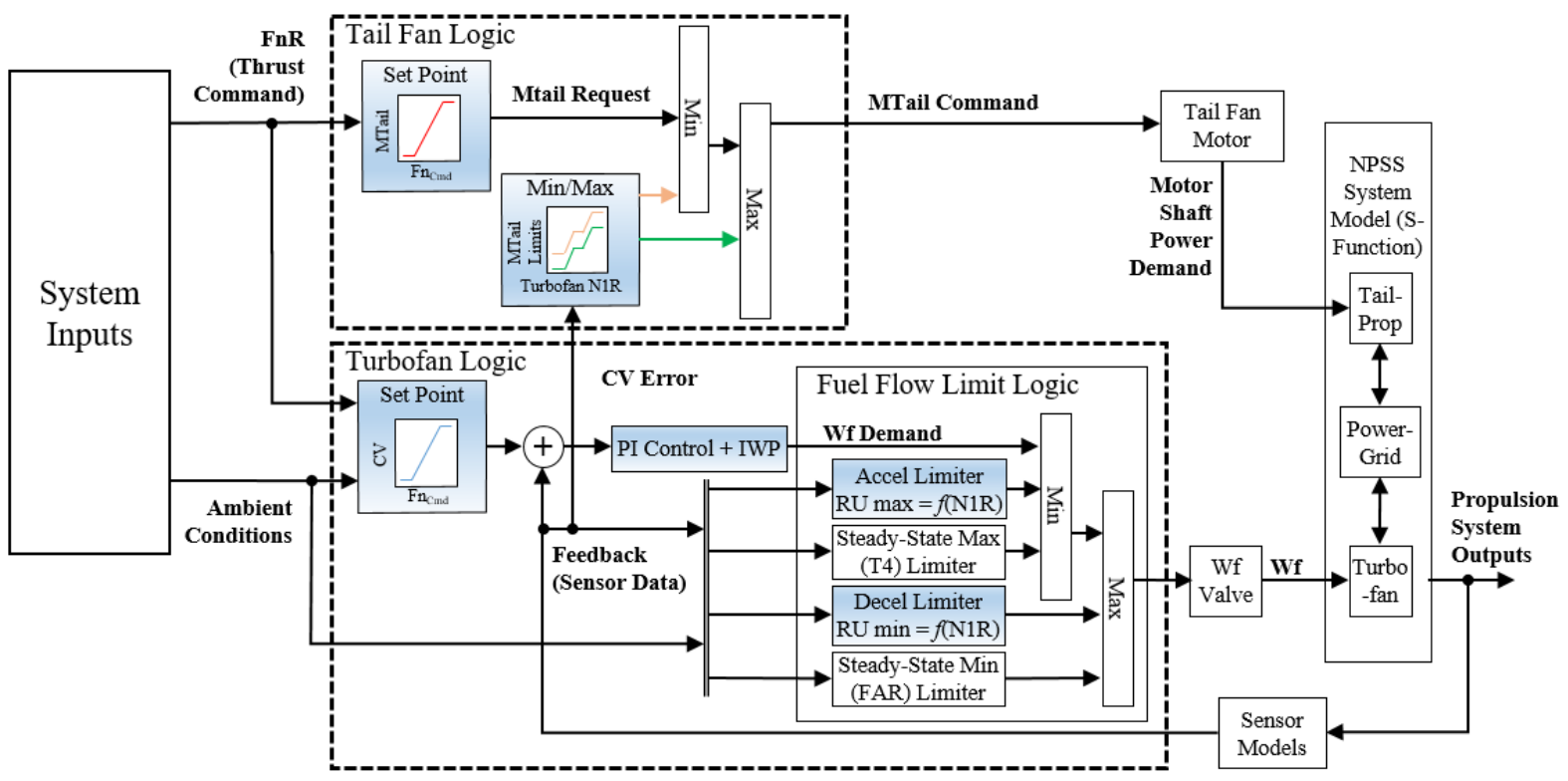

Fig. 5 Schematic of the turbofan and tail fan thrust/power control logic

a minimum fuel to air ratio (FAR) limiter. The ratio-unit is the ratio of the fuel flow to the static discharge pressure of the HPC (Ps3). It is a useful metric for identifying if the engine is accelerating or decelerating too quickly. The T4 limiter obviously keeps the engine from exceeding temperature limits, and the FAR limiter prevents the combustor from running too lean such that it could result in a flame out. Note that T4 and FAR are typically not directly measurable quantities and other less direct measurements are used to protect these limits. However, since this study was about demonstrating potential capabilities, it was sufficient as well as simpler to directly control these quantities. The limit controllers were PI controllers with IWP. IWP was important as it prevents the controllers from accumulating large amounts of error, particularly while the controller was inactive. The max-min logic selected the minimum of the fuel flow commands from the nominal controller and maximum limiters. It then compared the selected value with the maximum fuel flow commanded by the minimum limiters and selected the maximum fuel flow of those values.

The maximum RU limiter was the acceleration limiter and was of special consideration, particularly for the DSA studies provided later. Adjustments in the RU set-point schedule alter the responsiveness of the engine upon a request for an increase in power or thrust and in turn affect its operability. Of primary concern was exceeding the maximum T4 limit and minimum HPC SM limit. Note that the SM is a measure of how close the compressor is to stalling. During acceleration transients, the HPC SM typically decreases and undershoots the next steady-state HPC SM value to reach a local minimum before the engine settles into steady-state operation. As a result, additional HPC SM must be allotted in the engine design to accommodate such transients. This prompts conservativeness in the design that erodes potential efficiency and performance that the engine could theoretically possess. While slowing the engine transient down would reduce the undershoot in SM and improve the issue, this approach was constrained by a Federal Aviation Administration (FAA) requirement (Title 14, Chapter I, Subchapter C, Part 33, Subpart E, §33.73) [16] that states the following: (a) the engine must not surge, stall, exceed the maximum operating temperature, or experience any other detrimental factors while the engine is accelerated from minimum rated takeoff thrust to maximum thrust when the power control is moved from its minimum to maximum position in no more than $1 \mathrm{~s}$, and (b) the engine must be able to accelerate from its minimum flight idle power level, or from no more than $15 \%$ of the rated takeoff thrust, to $95 \%$ of the rated takeoff thrust within $5 \mathrm{~s}$. Therefore, the acceleration schedule has the arduous task of protecting the engine from potentially detrimental conditions while still allowing it to meet the $5 \mathrm{~s}$ response time requirement. In this application, the RU set-point schedule, otherwise called the acceleration schedule, was a function of the MN, Alt, and engine corrected fan speed (NfR). The TTECTrA maximum RU schedule design function creates the schedule. The function runs through numerous data points throughout the flight envelope and iteratively runs open-loop acceleration simulations in which the fuel flow was ramped up at varying rates until the prescribed transient operability margin constraints were just met (i.e. the minimum HPC SM limit or maximum T4 limit). The Wf/Ps3 data from those simulations are collected and used to construct the acceleration schedule.

The minimum RU limiter was the deceleration limiter. Its job was to protect the engine's fuel flow from decreasing too quickly such that it could cause operability issues. The typical issues that arise are reduced LPC or fan SM, resulting in stall, or a reduced FAR causing a potential flame out. The RU set-point schedule, or deceleration schedule, 
was created in a similar fashion as the acceleration schedule. Note that T4 and FAR set-points were single constant scalar values and therefore did not require the construction of set-point schedules.

In an actual application, the tail-cone thruster would probably be actively controlled, using the applied motor power as the input and the tail fan speed or estimated thrust as the control variable. The amount of power extracted from the underwing engines would be actively altered to appease the demand of the tail-cone thruster controller while also defending operability limits. However, in this study, the tail-cone thruster operated in an open-loop fashion where the power extracted from the engines and ultimately applied by the tail-cone motor was determined by the power schedule mentioned previously. The commanded tail power (MTail) was scheduled based on the MN, Alt, and commanded FnR. Since the commanded FnR can change instantaneously, it had the ability to cause large and abrupt changes in power extraction from the underwing engines that have the ability to negatively impact operability. This is true during large changes in the engine's commanded power or thrust. To mitigate this issue, limit logic was added to the motor controller logic. The limit logic specified maximum and minimum tail power commands that were scheduled based on the MN, Alt, and commanded FnR. The maximum and minimum tail power schedules were created by first constructing a nominal tail power schedule that relates the turbofan corrected fan speed to the tail power during steadystate operation. The maximum and minimum tail power schedules were simply set to be above or below $5 \%$ of this nominal schedule. The $5 \%$ value was chosen based on experience with prior simulations of the model that suggested that a 5\% deviation from nominal operation was the maximum tolerated before experiencing noticeable decreases in operability. A max-min controller structure, similar to what is applied in the limit logic of the fuel controller, was used to determined which signal gets passed to the motor.

The scheduled control logic applied to the VBV and VAFNs were derived from steady-state simulation of the NPSS model. As previously noted, the VAFN schedules were modified in an effort to reduce the maximum area of the VAFN and the overall range of variation.

The control approach applied and described here was not meant to be an optimal solution. There are more advanced control approaches that could be applied in order to obtain better transient performance and further promote operability and overall system stability and robustness. The control approach described here was utilized to demonstrate sufficient performance of the STARC-ABL concept without significant departure from conventional control architectures or approaches applied in the industry. If the concept is feasible and provides noticeable improvements with simple methods, then further enhancements are expected from more advanced techniques.

\section{TTECTrA Controller Results}

The operability limits applied in the controller design were set as follows: maximum $\mathrm{T} 4$ of $3400^{\circ} \mathrm{R}$, minimum HPC and LPC SM of 12\%, minimum fan and tail-fan SM of 10\%. To test the controller design, a Monte Carlo analysis was performed where the integrated model was run through burst and chop transients at random flight conditions and with random minimum and maximum corrected thrust set-points. The changes in thrust set-points were applied as a $1 \mathrm{~s}$ ramp. It was also assured that the analysis covered the data points at which the NPSS steady-state model was run for design and testing. This included crucial operating points such as takeoff, top of climb, and cruise. The Monte Carlo test points are given by Fig. 6. The red "+" symbols indicate flight envelope test cases used for design and testing, while the blue " $x$ " symbols indicate random test cases used for the rest of the Monte Carlo simulations.

The scatter plots shown in Fig. 7 indicate that the controller was able to maintain operability in all of the test cases and meets the FAA $5 \mathrm{~s}$ thrust response requirement for all scenarios where a takeoff or go-around is practical. It is evident by looking at Fig. 7a that the 95\% thrust time from idle to full power is well below $5 \mathrm{~s}$ for most of the test cases and all test cases below 15,000 ft. With the exception of two outliers, all test cases below 32,000 ft are able to make the takeoff thrust response time requirement. The thrust responses also exhibited little to no overshoot as indicated by Figure 7b. Most of the test cases had less than $1 \%$ overshoot while the maximum observed overshoot was less than $7 \%$. It was confirmed that the underwing engines were able to achieve $15 \%$ of their rated takeoff thrust or less in areas of the flight envelop that apply to the FAA thrust response requirement. As shown in Fig. 7c, the T4 limit does not appear to pose any issue at any of the flight conditions. The LPC and fan SMs plotted in Fig. 7e and 7f did not appear to pose an issue either, leaving significant amounts of excessive margin. The tail fan SM meets its $10 \%$

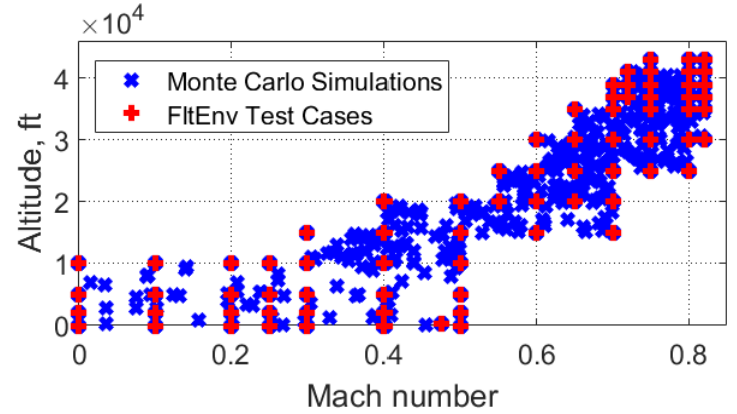

Fig. 6 Monte Carlo test points for the baseline controller 


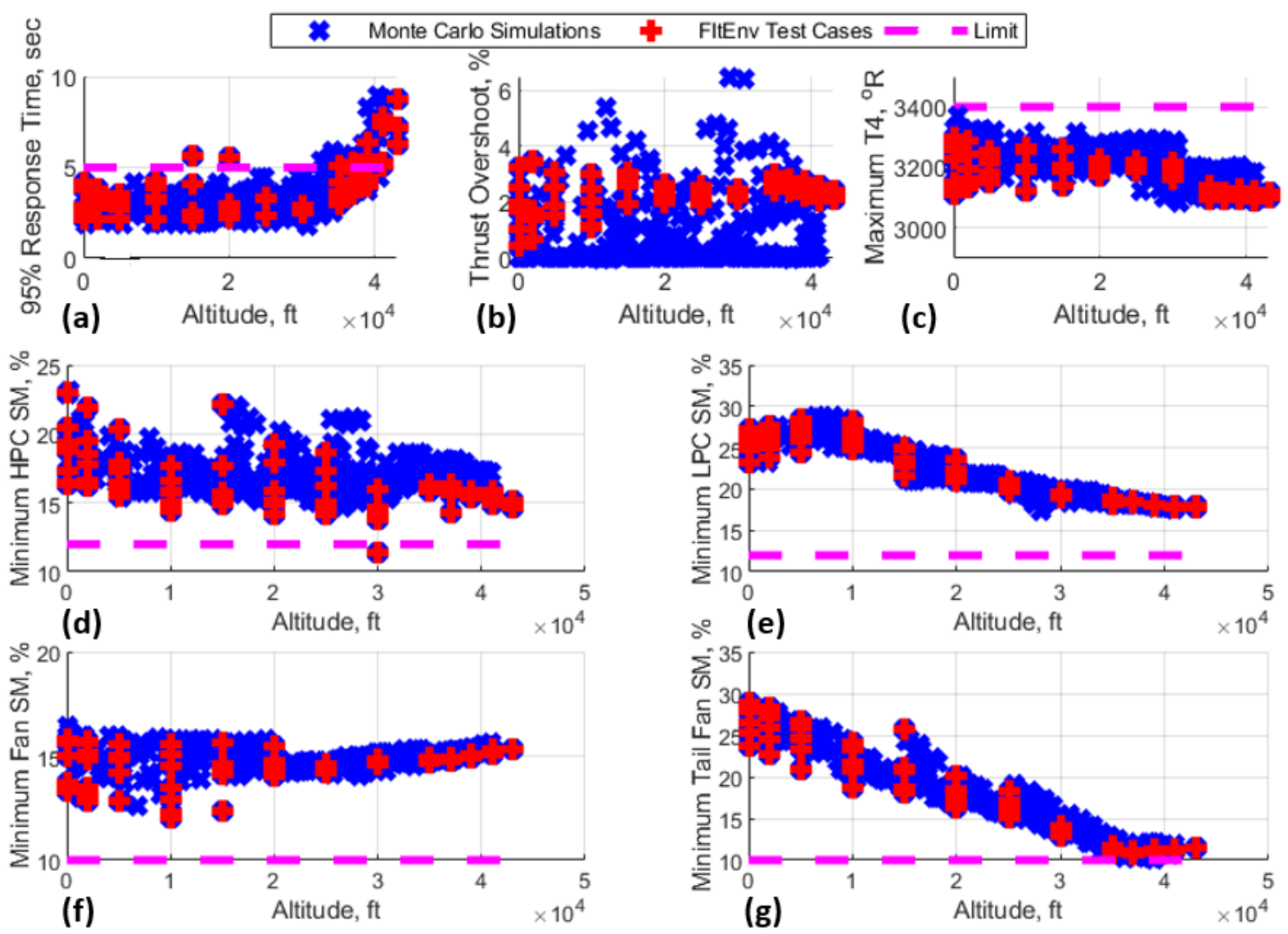

Fig. 7 Results from the Monte Carlo analysis

SM constraint at very high altitude ( $>35,000 \mathrm{ft}$ ), as shown in Fig. $7 \mathrm{~g}$, but does not violate it. In all but one test case the HPC SM, plotted in Fig. 7d, is respected with a significant amount of excess margin. The single point that defies the $12 \%$ SM limit had a value of $11.3 \%$ and occurs at $30,000 \mathrm{ft}$ and a Mach number of 0.8 . This test point could be improved in a few ways. One is to simply adjust the acceleration schedule to slow down the response and increase the 95\% thrust response time, which for this point is less than $3 \mathrm{~s}$. Slowing the response down to $5 \mathrm{~s}$ would undoubtedly improve the minimum SM and correct the issue. Also, given that this data point was at 30,000 $\mathrm{ft}$ where a takeoff or go-around scenario does not apply, the thrust response could conceivably be slowed down even more to remedy the issue if necessary. For these reasons, this outlier was not of concern. Based on the overall results, the controller was deemed sufficient. Furthermore, the STARC-ABL propulsion system was deemed feasible from an operability perspective to the extent that this study applies.

For a more complete look at the dynamic performance of the STARC-ABL propulsion system, some simulation data was also provided for evaluation in Fig. 8. Note that the thrust plotted in Fig. 8a is the net thrust of the entire propulsion system. The plots show the results of burst and chop profiles for four different test cases. In each test case the engine began at idle, accelerated to full power, and decelerated back to idle. The 4 test cases included SLS conditions (Alt $=0 \mathrm{ft}, \mathrm{MN}=0$ ), and flight conditions at 10,000 ft and Mach 0.4, 25,000 ft and Mach 0.8, and 41,000 $\mathrm{ft}$ and Mach 0.8. It was noted that the same trends observed in these plots were observed for other flight conditions. Trends in Fn, T4, FAR, and HPC SM were reminiscent of a typical turbofan engine. These trends are visible in Fig. 8a, 8f, 8g, and 8d respectively. However, some unusual trends are observed in the LPC SM, fan SM, and tail fan SM that are plotted in Fig. 8e, 8b, 8c respectively. While the LPC SM typically increases as the power increases, particularly when the engine was initially accelerating, the LPC SM was observed to decrease in this situation. Similar responses for the fan SM are shown. The reversal in trends was mainly attributed to significant power extraction from the low pressure spool.

First, consider the LPC SM. Its trend actually switches as the altitude and Mach number increase. The reason this occurred was power extraction coupled with the nature of the speed-based SM calculation from the LPC performance map. Before attempting to understand this, observe the simulation results on the LPC map shown in Figure 9. On the LPC map, pressure ratio (PR) is on the vertical axis, corrected flow $(\mathrm{Wc})$ is on the horizontal axis, each vertical curved 

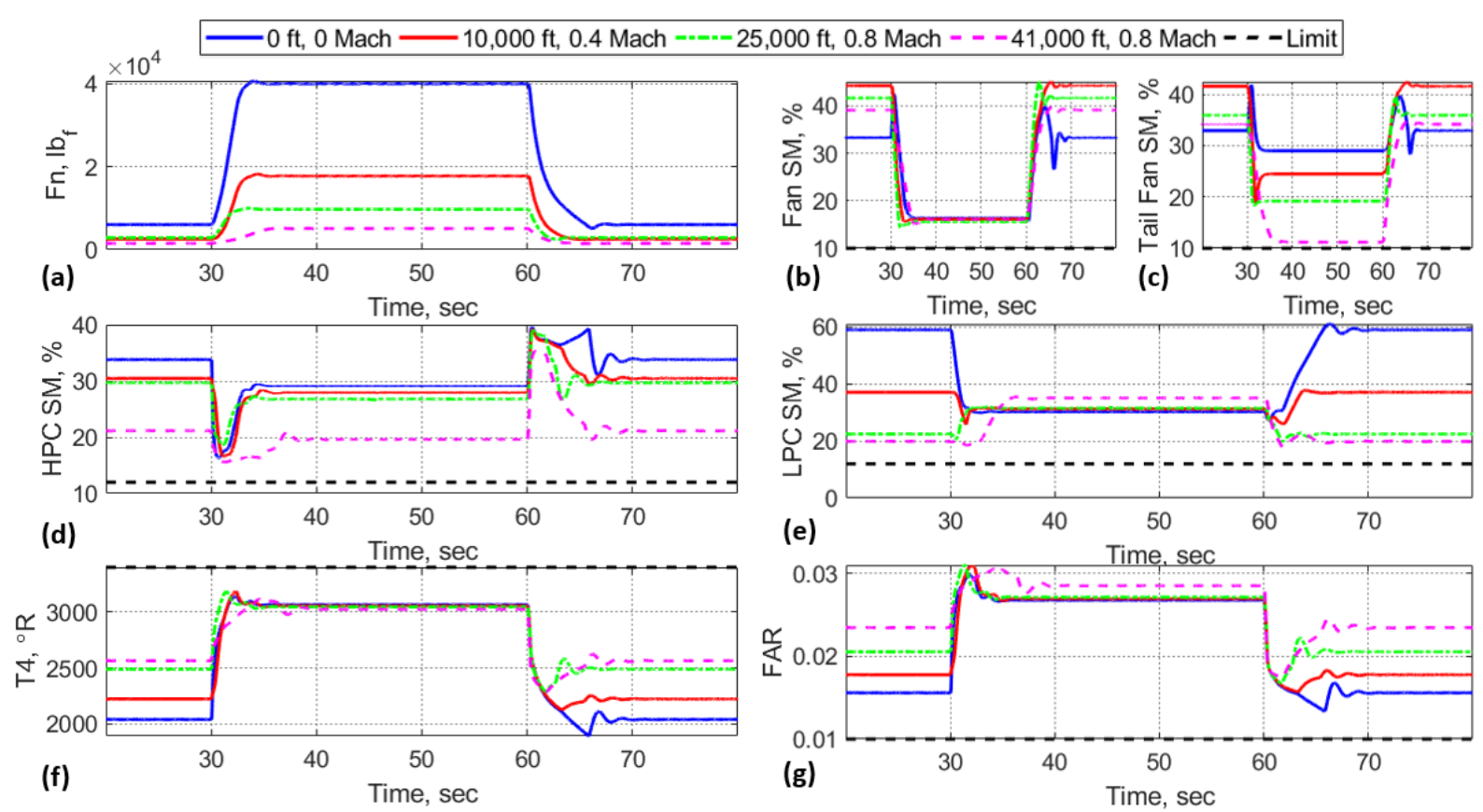

Fig. 8 Burst and chop simulation results for select cases

line represents a different corrected speed with lines increasing in speed from left to right, the horizontal curved lines and color map coordinates with the stall margin, and the bold black line indicates the stall line. Observe that at lower corrected speeds, the SM contours become compressed indicating that they are more sensitive to change. Furthermore, it was observed in Ref. [7] that increasing the shaft power tends to move the operating point on the LPC map toward the stall line. Extracting power from the shaft is expected to move the operating point away from the stall line. Ref. [7] also showed that movement of the operating point was more sensitive at lower corrected speeds. Figure 9 provides the normalized LPC performance map. The black circles distinguish the performance at the initial ground idle operating condition. It was evident that at low altitude and low Mach number, the engine was able to reach lower corrected speeds which, when combined with the observations above, explains the unusual trends observed for LPC SM at SLS and when at $10,000 \mathrm{ft}$ and a Mach number of 0.4. In particular, it described the large stall margin at low power. At the high altitude and Mach number conditions, it was observed that the minimum corrected speed increases significantly into a region where the SM was less sensitive to disturbances in shaft power and at this point, the effects of power extraction are harder to observe.

The fan SM had a consistent trend that persists throughout the flight envelope. The same observations made for the LPC map also apply to the fan map. However, power extraction tends to effect the fan differently than the LPC. Power extraction from the low pressure spool induces more fuel flow in order to increase the speed of the high pressure spool that

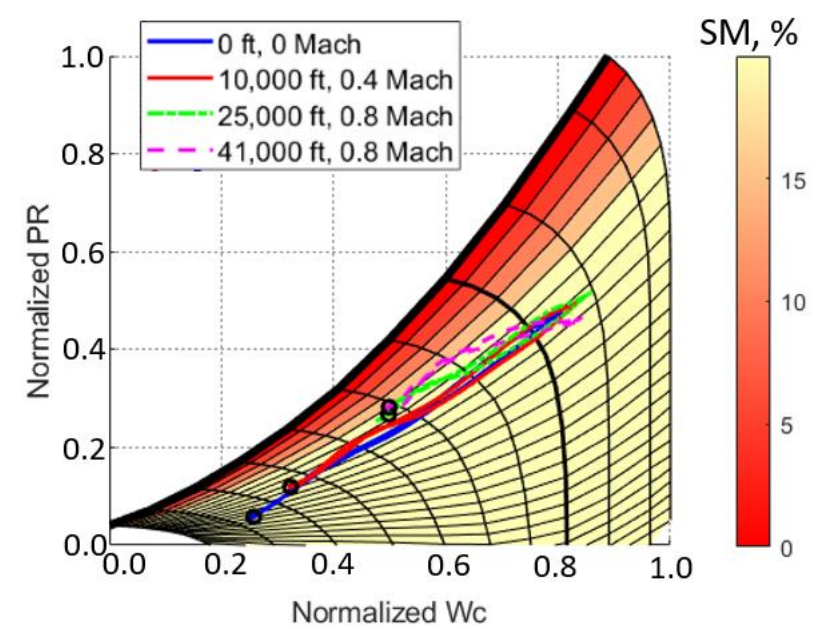

Fig. 9 Normalized LPC performance map supplies more power to the low pressure spool to satisfy the thrust demand. Increasing the speed of the high pressure spool tends to increase the airflow through the core. The increased flow, particularly at low speeds, tends to improve stall margin for the LPC. However, the increased airflow through the core tends to reduce the relative airflow that the fan blade was exposed to, and this results in offincidence flow for the fan which correlates to a reduced fan SM. As the power of the engine increases, so does the amount of power extraction. Due to the large amount of power extraction, the fan SM tends to decrease as the engine 
power increases. For the SLS case, it was observed that the power extraction is suppressing the natural fan SM response. This was evident by an initial increase in stall margin before it sharply decreases.

The tail fan SM had a consistent trend that was observed throughout the flight envelope. The operation of the tail fan was not tightly coupled to the operation of the turbofan cores in the same manner as the engine fans. In this case, the trends were attributed to the power schedule. Evidently, an increase in rotational speed demanded by the tail fan motor schedule was not matched with a proportional increase in airflow such that it can that maintain the same flow incidence. This tends to correlate to a reduced stall margin as the motor power increases.

Overall, the controller design protected the propulsion system from violating operability limits while it also achieved the responsiveness required to meet FAA requirements for transient operation. The control schedules for the VBV and VAFNs provided sufficient steady-state operability margin throughout the flight envelope, despite a significant reduction in the maximum area of the VAFNs compared to the original system design. Limit logic applied to the power requested by the tail fan motor prevented any detrimental reductions in operability during abrupt transients. Other operability limits, such as the maximum T4 limit, did not appear to pose an issue. The thrust responses also exhibited good behavior with little to no overshoot. To the extent of this study, the STARC-ABL system appears to be feasible from an operability perspective.

\section{Dynamic Systems Analysis Results}

The first subsection will detail dynamic system analysis of the STARC-ABL propulsion system, performed in a similar manner to Ref. [6] and [7]. The second subsection investigates the potential impact of adding energy storage to the STARC-ABL concept.

\section{A. Performance vs. Operability Trade}

Having demonstrated the operability of the STARC-ABL propulsion system design throughout its flight envelope, the focus was to identify any excessive operability margins that could be limiting the engine's performance. If the margins are deemed conservative, then the engine design constraints can be relaxed. This opens up the engine design space to consider options such as having a more highly loaded compression system with fewer stages. With the capabilities of controls considered, the engine can operate closer to its design conditions, thus enabling a more efficient design to be achieved.

The same control design process was applied in the dynamic systems analysis studies as was described prior for the baseline controller. The difference was that the operability design constraints were varied in the controller design. Primarily the acceleration limit logic was designed for five different minimum HPC SM design values. The engine health parameters were also varied to build confidence that the control design would be sufficient throughout the lifespan of the propulsion system.

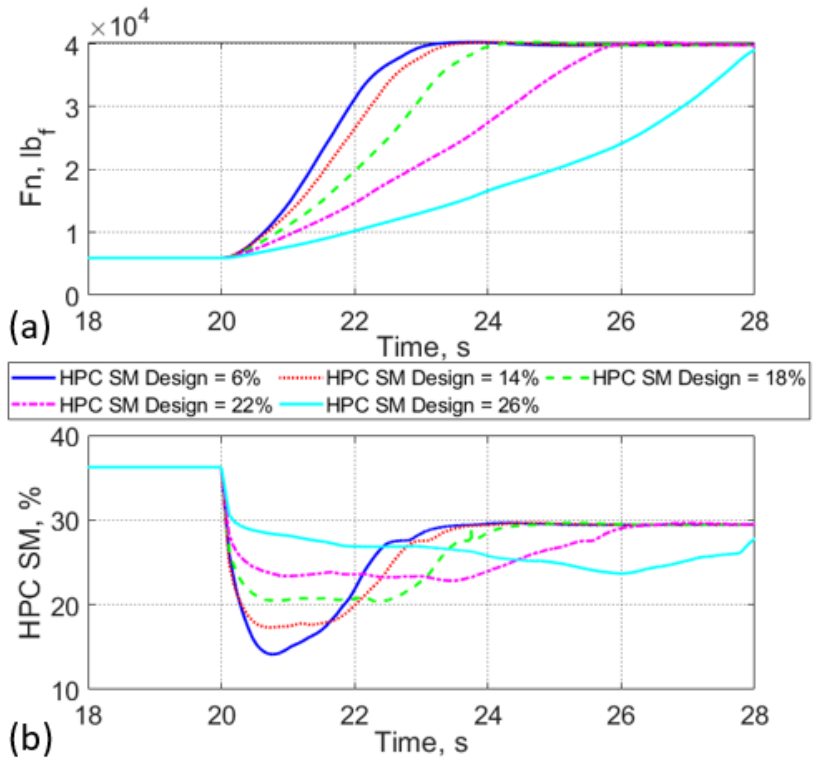

Fig. 10 Thrust and HPC SM response for a burst and chop simulation

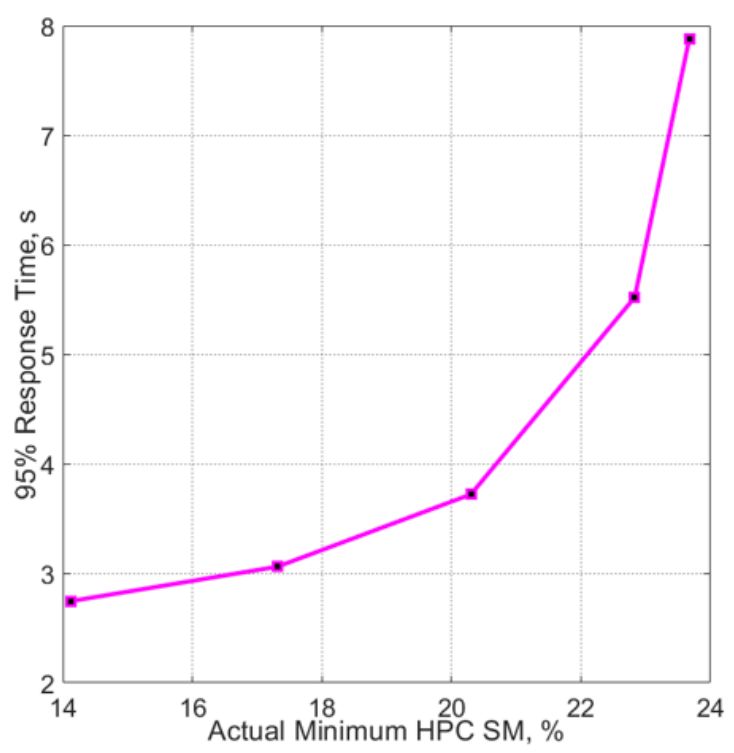

Fig. 11 Response time vs. HPC operability for an SLS 


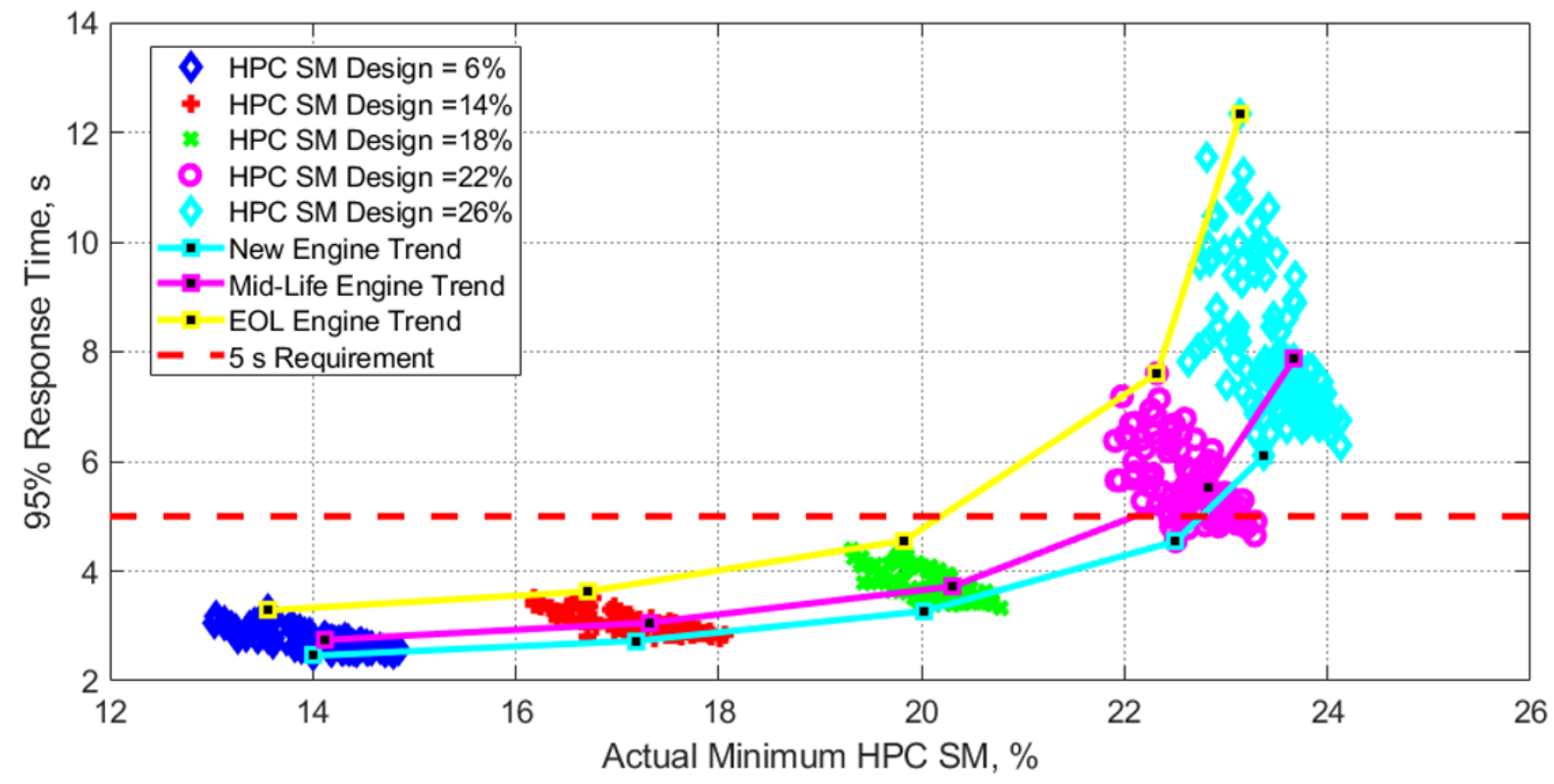

Fig. 12 Response time vs. HPC SM at SLS with health parameter variations

Comparison of the different acceleration schedules were made based on their performance when applied to an idle to full power transient at SLS on a hot day $\left(27^{\circ} \mathrm{R}\right.$ above the standard atmospheric temperature). The net thrust and HPC SM results are shown in Fig. 10a and 10b, respectively. The 95\% response time vs. the minimum HPC SM is shown in Fig. 11. These results suggest that controller designs using a minimum HPC stall margin of $18 \%$ or less was able to meet the FAA requirement for thrust responsiveness. The results were for a mid-life system but the controller should be designed to provide acceptable operability and performance throughout its lifespan. Therefore, Monte Carlo simulations were run in which the system health parameters were randomly varied. The results are shown in Fig. 12 and indicate that the controller designed to protect 18\% HPC SM protects operability limits and meets the FAA response time requirement. The minimum HPC SM was roughly $19 \%$. Theoretically, this allows for a $7 \%$ reduction in HPC SM while still protecting the $12 \%$ limit. However, because the $5 \mathrm{~s} 95 \%$ response time requirement will apply to flight conditions other than SLS, a more conservative reduction of 3\% HPC SM was recommended without a comprehensive analysis over the range of all relevant flight conditions. With the additional margin, the turbomachinery could potentially be designed to enable a more efficient engine that ultimately results in reduced fuel burn.

\section{B. Energy Storage Study}

The study of energy storage is inspired by Ref. [3], which explores how hybrid electric architecture could be utilized to improve the operability and performance of the turbomachinery. STARC-ABL does not have energy storage in its nominal system design. But what if it did? If STARC-ABL had a battery, super-capacitor, and or any other means of supplying or absorbing energy, it was hypothesized that operability benefits could be gained. One way to exploit energy storage is to use it to reduce the coupling between the tail-cone thruster and underwing engines. In this study, supplementing power to the tail cone thruster or reducing power extraction from the underwing engines during steady-state operation was not considered. This decision was made to avoid affecting steady-state performance and inducing the need to make major changes to the propulsion system design. However, energy storage was considered for use during transients. In this study, the tail-cone was assumed to maintain its nominal operation, but the origins of the power used to drive the motor may differ.

First, consider an acceleration transient. By supplying a fraction of the power demanded by the tail-cone motor with an energy storage device (ESD), the workload on the engine can be alleviated. Less power extraction from the low pressure spool means that the combustor and high pressure spool will not have to work as hard to maintain the speed of the low pressure spool to achieve the desired thrust. This results in a more gradual increase in fuel flow and a slower initial acceleration of the high pressure spool. This in turn correlates to an increase in the minimum HPC SM. Since the low pressure spool will respond faster with less power extraction, the acceleration schedule was adjusted to achieve the same response time that it had with the nominal amount of power extraction. This should enable further improvements in operability. Also, if the baseline design responds faster than the $5 \mathrm{~s}$ requirement then there was room for additional operability benefits. 
During decelerations transients, more power extraction was allowed from the low pressure spool. The excess power that was not used by the tail cone motor could potentially be absorbed by the ESDs. The increase in power extraction from the low pressure spool improves the LPC operability as was demonstrated in Ref. [3].

The purpose of this study was to begin investigating the impact of utilizing ESDs to improve transient operability, not to find the optimal way to implement it. To investigate the proposed theory, a simple ad-hoc control approach was utilized. The fraction of power supplied to the tail motor by the ESD was scheduled. The rest of the power was supplied by the engines. The fraction of power supplied or absorbed by the ESDs was scheduled based on the normalized error of the active control variable. When the error is high, the fraction of power supplied by the ESD is set to a given value. When the error is low, the power supplied by the ESD tapers off to zero to assure that the ESDs do not supply or absorb power during steady-state operation. The normalized error is the dimensional error of the active control variable normalized by the

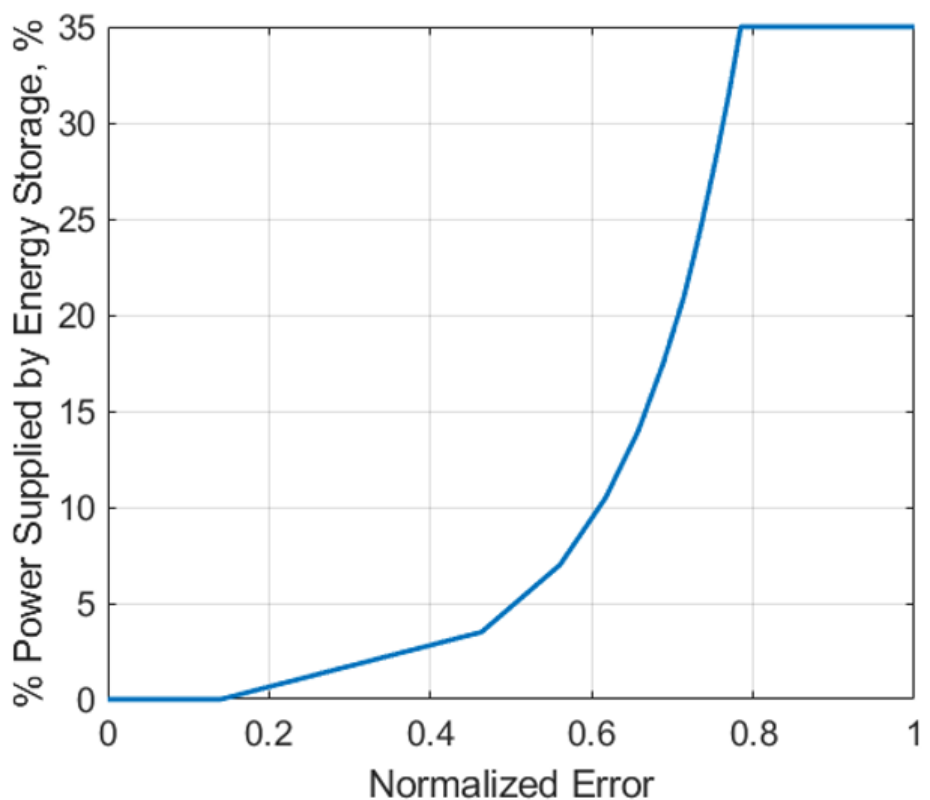

Fig. 13 Energy storage device power faction schedule expected range of variation of that controlled variable. An example of the schedule is provided by Fig. 13. In this example, the ESD supplies $35 \%$ of the demanded power when the normalized error was greater than $\sim 0.78$. Various schedules were applied in simulation with different levels of energy storage assistance. The schedules were also compressed or stretched by different degrees to make the transition between transient and steady-state operation more abrupt or gradual.

To test the concept, the model was run through a chop and burst scenario at the SLS condition, changing between idle and max power conditions. Four different cases were simulated: (1) the baseline propulsion system and controller, (2) an ESD assisted propulsion system with the same rise time (Tr) as the baseline system, (3) the baseline system with a modified acceleration schedule set to just meet the $5 \mathrm{~s}$ rise time requirement, and (4) an ESD assisted propulsion system with a $5 \mathrm{~s}$ rise time. Note that the tail motor limit logic was ignored in the simulations that utilized assistance from the ESDs as it was no longer necessary. Using the schedule in Fig. 13, the results shown in Fig. 14 were observed. The power supplied or absorbed by the ESDs is quite significant as shown in Fig. 14a. The augmentation had little effect on the motor power seen in Fig. 14c. However, it slowed down the response of the generator power extraction and it required an increase in power extraction during the deceleration transient as shown in Fig. 14b. The impact was a slight increase in stall margins during the transients which can be viewed in Fig. 14d and 14e. Figure 15a and 15b show the HPC SM and LPC SM variation during the acceleration and deceleration transients respectively. Application of the schedule yielded a $0.5 \%$ improvement in HPC SM with the same rise time as the baseline model. If the response were slowed down to just meet the $5 \mathrm{~s}$ requirement, then application of the schedule yields a $6.5 \%$ improvement over the baseline model and a $1.2 \%$ improvement over the baseline model with a the same response time. During the deceleration, the initial dip in LPC SM was reduced by $\sim 1 \%$ as a result of the initial increase in power extraction. The drawback to this approach was that it would require a larger generator, or that the $2000 \mathrm{hp}$ generators be able to achieve the additional power extraction for the short duration of the transients.

Other power schedules were applied in the simulation. It was noted through evaluation of those test cases that the manner in which power extraction from the underwing engines is altered during the transient was important. If the reduction in power extraction is large and abrupt, the tendency is for the minimum HPC SM to increase. However, the minimum LPC SM is reduced at the start of the transient. As the power schedules become more gradual and the load on the ESDs is reduced, the HPC SM benefits decrease but the LPC SM issue was improved and eventually subsides. Thus, any modifications to the control of the generators must balance the operability of all of the components. As mentioned before, the control approach considered in this investigation was far from optimal. However, it does imply the prospect of obtaining potential benefits through the addition of ESDs. It is clear that the power management should be handled more intelligently to achieve better results. The idea proposed here could be merged with a more 
intelligent approach to managing the energy in the propulsion system such as utilizing the techniques encompassed by the Turbine Electrified Energy Management (TEEM) concept [3]. The addition of an electric machine or dual use of an electric starter motor would also be beneficial, as was demonstrated in Ref. [3]. By combining all of these ideas, it is theoretically possible to remove all of the transient operability margin allowing the engine design space to be significantly expanded.
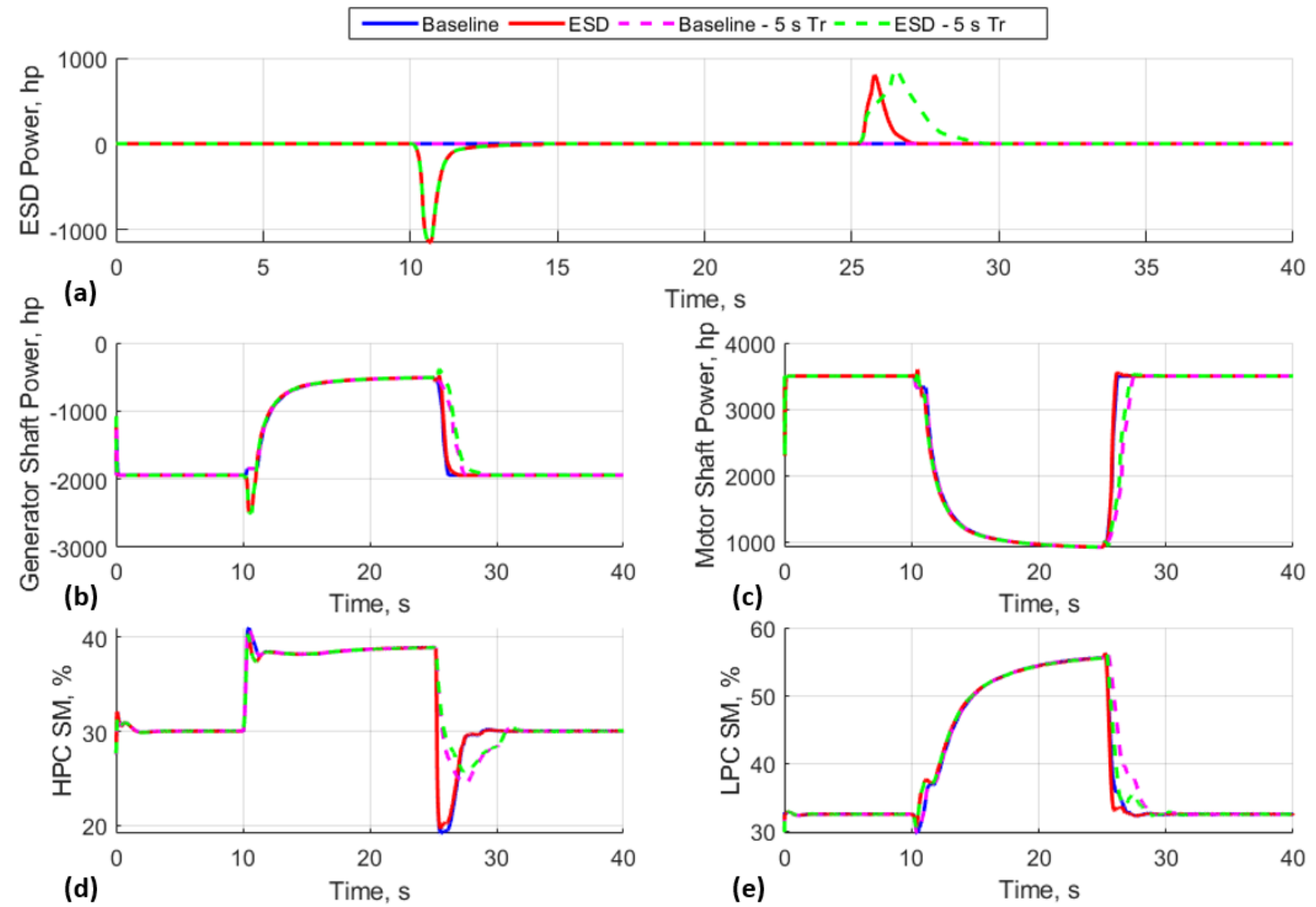

Fig. 14 Energy storage study results with $35 \%$ power assistance from the energy storage device

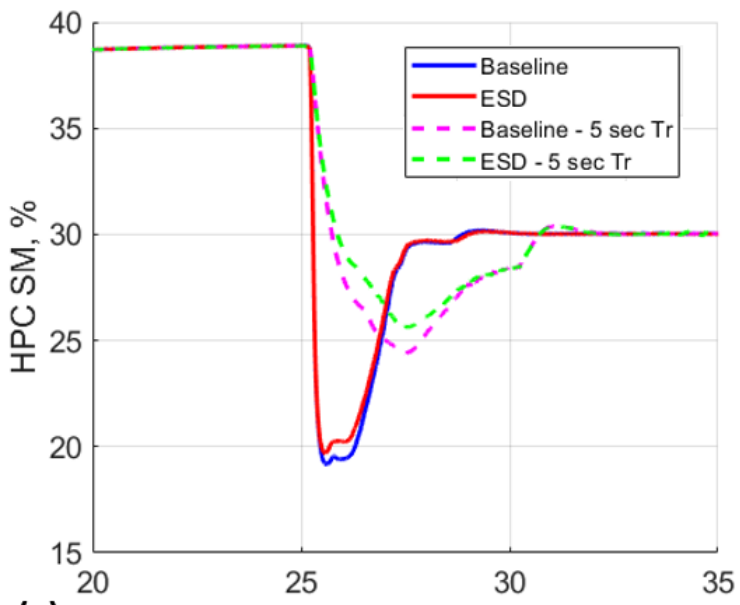

(a)

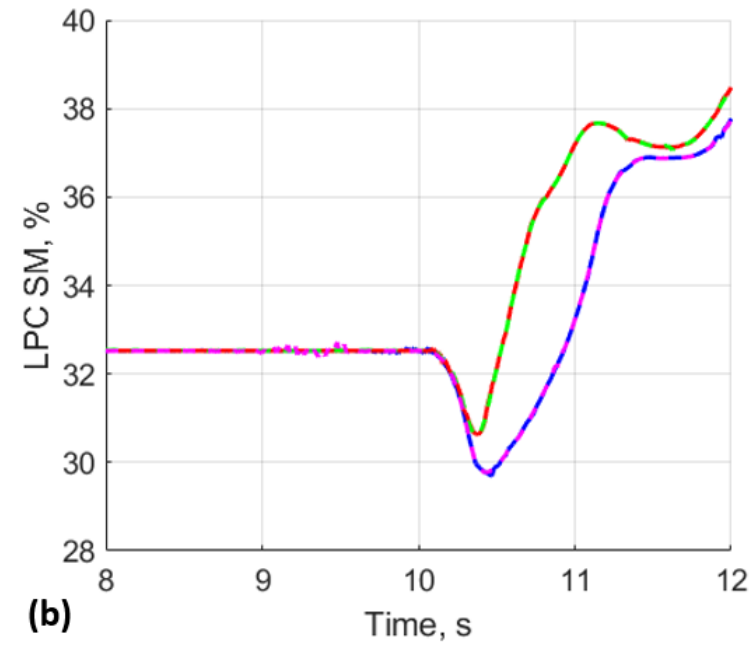

(b)

Time, s

Fig. 15 HPC SM plotted for the acceleration transient (a), and LPC SM plotted for the deceleration transient (b) 


\section{Conclusion}

The Single-aisle Turboelectric AiRCraft with Aft Boundary Layer propulsor (STARC-ABL) concept was evaluated from the perspective of the operability of its propulsion system. The propulsion system was implemented in simulation as a nonlinear dynamic model that included actuator and sensor dynamics. The control architecture utilized active and scheduled control logic to maintain operability of the STARC-ABL propulsion system throughout the flight envelope. Limit control logic was utilized to ensure operability limits were maintained while still meeting transient performance requirements. Altogether, the control scheme employed was demonstrated to maintain sufficient operability margins in steady-state and through transients such that the concept was deemed feasible. However, it should be noted that this study did not consider fault analysis and should not be considered a comprehensive evaluation of operability. Approximately $3 \%$ of excessive HPC stall margin was identified through dynamic systems analysis (DSA) studies of the STARC-ABL propulsion system. The excessive operability margin could be leveraged in an engine re-design to achieve benefits for the engine. A study to investigate the effect of adding energy storage to the STARC-ABL concept revealed the potential to leverage stored electrical energy to reduce the coupling between the underwing engines and the tail-cone thruster during transients. This could further improve operability and potentially widen the engine design space more than was previously identified.

\section{Acknowledgments}

The authors would like to acknowledge support for this work provided by the Advanced Air Transportation Technologies (AATT) project under the NASA Aeronautics Research Mission Directorate (ARMD). The authors would also like to acknowledge those within NASA, James Felder, William Haller, and Thomas Lavelle, who developed the STARC-ABL NPSS model, helped to modify it for these studies, and provided guidance to the work.

\section{References}

[1] Ashcraft, S.W., Padron, A.S., Pascioni, K.A, Stout Jr., G.W., and Huff, D.L., "Review of Propulsion Technologies for N+3 Subsonic Vehicle Concepts," NASA TM 2011-217239, October, 2011.

[2] Hathaway, M.D., Del Rosario, R., and Madavan, N.K., "NASA Fixed Wing Project Propulsion Research and Technology Development Activities to Reduce Thrust Specific Energy Consumption," NASA TM 2013-216548, July 2013.

[3] Culley, D., Kratz, J., and Thomas, G., "Turbine Electrified Energy Management (TEEM) For Enabling More Efficient Engine Designs," AIAA Propulsion \& Energy Forum, Cincinnati, OH. 2018.

[4] Welstead, J., and Felder, J., "Conceptual Design of a Single-Aisle Turboelectric Commercial Transport with Fuselage Boundary Layer Ingestion," 54th AIAA Aerospace Sciences Meeting, San Diego, CA. 2016.

[5] Bowman, C., Felder, J., and Marien, T., "Turbo- and Hybrid-Electrified Aircraft Propulsion Concepts for Commercial Transport," AIAA/IEEE Electric Aircraft Technologies Symposium, Cincinnati, OH. 2018.

[6] Csank, J.T., and Thomas, G.L., "Dynamic Analysis for a Geared Turbofan Engine with Variable Area Fan Nozzle," AIAA 2017-4819, 53 ${ }^{\text {rd }}$ AIAA/ASME/SAE/ASEE Joint Propulsion Conference, Atlanta, GA, July 10-12, 2014.

[7] Thomas, G., Culley, D., Kratz, J., and Fisher, K., "Dynamic Analysis of the hFan, a Parallel Hybrid Electric Turbofan Engine," AIAA Propulsion \& Energy Forum, Cincinnati, OH. 2018.

[8] Connolly, J., Chapman, J., Stalcup, E., Hunker, K., Chicatelli, A., and Thomas, G., "Modeling and Control Design for a Turboelectric Single Aisle Aircraft Propulsion System," AIAA/IEEE Electric Aircraft Technologies Symposium, Cincinnati, OH. 2018.

[9] Welstead, J., Felder, J., Guynn, M., Haller, W., Tong, M., Jones, S., Ordaz, I., Quinlan, J., and Mason, B., "Overview of the NASA STARC-ABL (Rev. B) Advanced Concept," https://ntrs.nasa.gov/search.jsp?R=2017 0005612\&hterms $=$ STARC-ABL\&qs=N\%3D0\%26Ntk\%3DAll\%26Ntt\%3DSTARCABL\%26Ntx\%3Dmode\%2 520 matchallpartial, [retrieved January, 2019].

[10] The Ohio Aerospace Institute, on behalf of the NPSS Consortium, "NPSS User Guide," 2010.

[11] Tong, M., and Naylor, B., "An Object-Oriented Computer Code for Aircraft Engine Weight Estimation," GT2008-50062, ASME Turbo Expo 2008, Berlin, Germany, July 9-13, 2008.

[12] Claus, R.W., Evans, A.L., Lylte, J.K. and Nichols, L.D., "Numerical Propulsion System Simulation,” Computing Systems in Engineering, Vol 2, No. 4, pp 357-364, 1991.

[13] Chin, J., Csank, J., Haller, W., and Seidel, J., "An Introduction to Transient Engine Applications Using the Numerical Propulsion System Simulation (NPSS) and MATLAB®,” NASA TM 2016-218922, January, 2016. 
[14] May, R.D., Csank, J., Lavelle, T.M., Litt, J. S., and Guo, T.-H., "A High-Fidelity Simulation of a Generic Commercial Aircraft Engine and Controller," Proceedings of the 46th AIAA/ASME/SAE/ASEE Joint Propulsion Conference, AIAA-2010- 6630, Nashville, TN, July 2010.

[15] Adibhatla, S., Ding, J., Garg, S., Griffith, S., Kranofski, K., Payne, N., Simon, D., and Wood, B., "Propulsion Control Technology Development Needs to Address NASA Aeronautics Research Mission Goals for Thrusts 3a and 4," AIAA 2018-4732, AIAA Propulsion \& Energy Forum, Cincinnati, OH. 2018.

[16] Federal Aviation Administration, "Title 14 of the Code of Federal Regulations", https://www.ecfr.gov/cgibin/text-idx?SID=2df09c833c6cae09d18ba3a5ae91abb0\&mc=true\&node=se14.1.33_173\&rgn=div8, [retrieved January, 2019]. 